\title{
Uterine Carcinosarcoma Confined to the Pelvis: A Retrospective Review and Outcome Analysis
}

\author{
Hualei Li, ${ }^{1}$ Mindi J. TenNapel, ${ }^{1}$ Amina Ahmed, ${ }^{2}$ Lilie Lin, ${ }^{3}$ \\ Sudershan K. Bhatia, ${ }^{1}$ and Geraldine Jacobson ${ }^{4}$ \\ ${ }^{1}$ Department of Radiation Oncology, University of Iowa Hospitals and Clinics, 200 Hawkins Drive, Iowa City, IA 52242, USA \\ ${ }^{2}$ Department of Obstetrics and Gynecology, University of Iowa Hospitals and Clinics, 200 Hawkins Drive, Iowa City, IA 52242, USA \\ ${ }^{3}$ Department of Radiation Oncology, Hospital of the University of Pennsylvania, 3400 Civic Center Boulevard, PCAM/TRC 4 West, \\ Philadelphia, PA 19104, USA \\ ${ }^{4}$ Department of Radiation Oncology, West Virginia University Healthcare, 1 Medical Center Drive, Morgantown, WV 26506, USA
}

Correspondence should be addressed to Hualei Li; hualei-li@uiowa.edu

Received 8 October 2013; Accepted 1 February 2014; Published 11 March 2014

Academic Editor: Zsuzsanna Kahán

Copyright (C) 2014 Hualei Li et al. This is an open access article distributed under the Creative Commons Attribution License, which permits unrestricted use, distribution, and reproduction in any medium, provided the original work is properly cited.

Objective. We compared the treatments of uterine carcinosarcoma at our institution and evaluated their impact on survival. Methods. A retrospective analysis was performed on 60 eligible patients with carcinosarcoma limited to the pelvis. Subjects were divided into four categories: surgery, surgery plus chemotherapy, surgery plus radiation therapy, and a combination of surgery, chemotherapy, and RT. The most commonly used chemotherapy was cisplatin and/or carboplatin and taxol. Radiotherapy included external beam radiation therapy (EBRT) alone or with high dose rate (HDR) brachytherapy or HDR brachytherapy alone. Survival probability data were computed using the Kaplan-Meier method. The differences between groups were compared using the log-rank test. Results. The combination of surgery and radiation therapy with or without chemotherapy is seen to improve overall survival (OS) compared to surgery alone ( $P=0.044$ and $P=0.028$, resp.). Brachytherapy involving three HDR vaginal cylinder fractions shows an equally effective reduction in local recurrence compared to EBRT. Conclusion. Our study of a relatively large number of carcinosarcoma patients suggests that adjuvant radiation therapy improves OS compared to surgery alone. Brachytherapy with 3 HDR vaginal cylinder fractions is preferred because of its time-saving, better tolerance, low toxicity and equivalent OS, and local control compared to EBRT.

\section{Introduction}

Carcinosarcoma is a rare malignant tumor composed of both epithelial (carcinomatous) and mesenchymal (sarcomatous) components [1]. In Western populations, uterine carcinosarcomas account for less than $5 \%$ of all cancers of the uterine corpus [2]. It usually occurs in postmenopausal women more than 60 years old [3]. Approximately $35 \%$ of carcinosarcoma patients present with advanced-stage disease at diagnosis. The five-year overall survival rate for all stages is $33-39 \%$ due to the high propensity for both local and distant relapse [46]. For patients with stage I disease, overall 5-year survival is approximately $50 \%$, and for patients with advanced-stage disease, 5 -year overall survival is less than 25\% [7]. Cancer stage, particularly, depth of myometrial invasion, is the most important prognostic factor in carcinosarcoma $[7,8]$.

Although surgery is the primary treatment, several retrospective studies were performed to determine which adjuvant therapy is best: chemotherapy and/or radiotherapy [3]. Due to high local and distant recurrence rates, radiotherapy and chemotherapy have been used to increase the probability of local or distant control and several studies have demonstrated decreased rates of local and distant failure with chemoradiotherapy or chemotherapy and radiotherapy alone [6, 9-11]. However, few studies have demonstrated any improvement in progression- free and overall survival $[12,13]$.

At our institution, we treated a comparatively large number of uterine carcinosarcoma patients between 2000 and 2011 
despite its rarity. Management of this cancer included surgery alone, surgery plus chemotherapy, surgery plus radiation therapy, and a combination of surgery, chemotherapy, and radiation therapy. In 2005, our institution replaced "pelvic RT and/or chemotherapy" with "high dose rate (HDR) vaginal brachytherapy plus chemotherapy" as the preferred treatment of carcinosarcoma patients based on our clinical experience and reported evidence. In this study, we review our experience of treating carcinosarcoma patients and report the results of local control, distant metastasis control, and overall survival rate.

\section{Materials and Methods}

2.1. Patient Population. After the approval of our institutional review board, we reviewed all medical records of patients who were diagnosed with uterine carcinosarcoma from January 2000 to November 2011. There were a total of 83 patients. Three were excluded due to incomplete treatment history. A further 11 patients with distant metastases were excluded. Three patients who declined surgery were excluded and another two died before surgery. Four patients presented a different final pathology although the initial biopsy showed carcinosarcoma. The remaining 60 patients with stage IIII disease limited to pelvis were included in this study. All patients staged using legacy FIGO stages were restaged using the FIGO 2010 standard based on their pathology reports. The mean age of these patients was 65 years (range 39-86). The time of diagnosis was considered to be the pathology report date of either biopsy or surgical procedure, whichever was earlier. The average follow-up length was 34 months (range 1 to 124). Time from diagnosis to recurrence and death or latest contact was also calculated.

2.2. Treatment. All patients underwent surgery. The most commonly used chemotherapy protocol at our institution was concurrent cisplatin every 3 weeks with radiation, followed by carboplatin and taxol (3-6 cycles, carboplatin AUC = 5 and taxol $175 \mathrm{mg} / \mathrm{m}^{2-}$ ). Radiotherapy included external beam radiation therapy (EBRT) to the whole pelvis using 10$18 \mathrm{MeV}$ photons generated by a linear accelerator and HDR vaginal cylinder brachytherapy was performed using HDR Iridium 192 after-loading technique. Radiation treatment modalities included EBRT alone, vaginal cuff brachytherapy alone, and EBRT plus brachytherapy. Before 2005, the most commonly used radiation therapy regimen was EBRT to the pelvis using a four-field box technique (45-50.4 Gy) with or without HDR brachytherapy using Ir192 (usually 6-7Gy in one fraction, only one patient received $6 \mathrm{~Gy} \times 2$ ). After 2005 brachytherapy was the preferred treatment choice with 1518 Gy delivered in 3 fractions in most cases. The dose was prescribed to $5 \mathrm{~mm}$ beyond the cylinder surface. One patient who received both chemotherapy and radiotherapy received only 27 Gy EBRT due to acute radiation induced enteritis and small bowel obstruction.

2.3. Statistical Methods. Chi-square test, Fisher's exact test, or Student's $t$-test as appropriate, was utilized to determine the difference between the treatment groups. Survival probability data were computed using the Kaplan-Meier method, and the differences between groups were compared using the log-rank test. The overall survival (OS), time to local recurrence (TLC), and time to metastasis (TTM) were computed and compared.

\section{Results}

Median followup for all surviving patients was 3.61 years (with a range of $0.477-10.35$ ) with an average follow-up time of 2.8 years for all patients included in this study. Median age at diagnosis was 65.5 years old. Median time from diagnosis to surgery is 24 days (range 0-179). Only two patients underwent surgery beyond 2 months after diagnosis. The delay in these two cases was for patient's personal reasons.

Table 1 lists the characteristics of these 60 patients. Table 2 lists the characteristics of 32 patients who received radiation therapy.

Forty-five patients had total abdominal hysterectomy and bilateral salpingoophorectomy, (TAH-BSO) and pelvic and paraaortic lymph node dissection (PPLND); 8 patients had TAH-BSO; 7 had TAH-BSO and pelvic lymph node dissection (PLND). The surgical distribution appears not to be different among 4 groups $(P=0.15)$. Statistical analysis revealed no overall survival difference when comparing TAH-BSO to TAH-BSO and LND $(P=0.88)$ in our study. Twenty-one patients were treated with surgery alone, 27 patients received chemotherapy, and 32 patients underwent radiation therapy at the treating physician's discretion. Only 7 patients received chemotherapy alone as adjuvant therapy. In surgery only group, 2 patients underwent TAH-BSO PLND and optimal cytoreduction. In surgery plus chemotherapy group, 1 patient underwent TAH-BSO and optimal cytoreduction. In surgery plus RT group, none underwent optimal cytoreduction. In surgery plus chemotherapy and radiotherapy group, 1 patient underwent TAH-BSO PPLND and optimal cytoreduction.

Statistical differences were tested between different treatment groups without further stratification by age and stage. Age and stage variations were tested between every two groups. Age analyses showed that surgery alone included older patients than the patient group who received surgery plus chemotherapy plus radiotherapy $(P=0.041)$. No age differences were detected in other group to group comparisons: surgery alone versus surgery plus chemotherapy $(P=0.99)$, surgery alone versus surgery plus RT $(P=0.81)$, surgery plus chemotherapy versus surgery plus RT plus chemotherapy $(P=0.08)$, and surgery plus chemotherapy versus surgery plus RT $(P=0.84)$. Age analyses for the radiation groups were insignificant: brachytherapy only versus brachytherapy and EBRT $(P=0.59)$, EBRT only versus brachytherapy and EBRT $(P=0.34)$, and EBRT only versus brachytherapy only $(P=0.65)$.

Stage distribution difference analyses showed that more stage II patients were included in the surgery plus RT group than compared to the group of all three treatments combined $(P=0.01)$. Global tests showed no difference between the distribution of stage I $(P=0.94)$ or stage III $(P=0.18)$. 
TABLE 1: Patients' characteristics in each group and their corresponding TLR (time to local recurrence), TTM (time to metastasis), and overall survival. To be included in range, patients have to have an event (such as local recurrence, distant metastasis, or death) in the corresponding category.

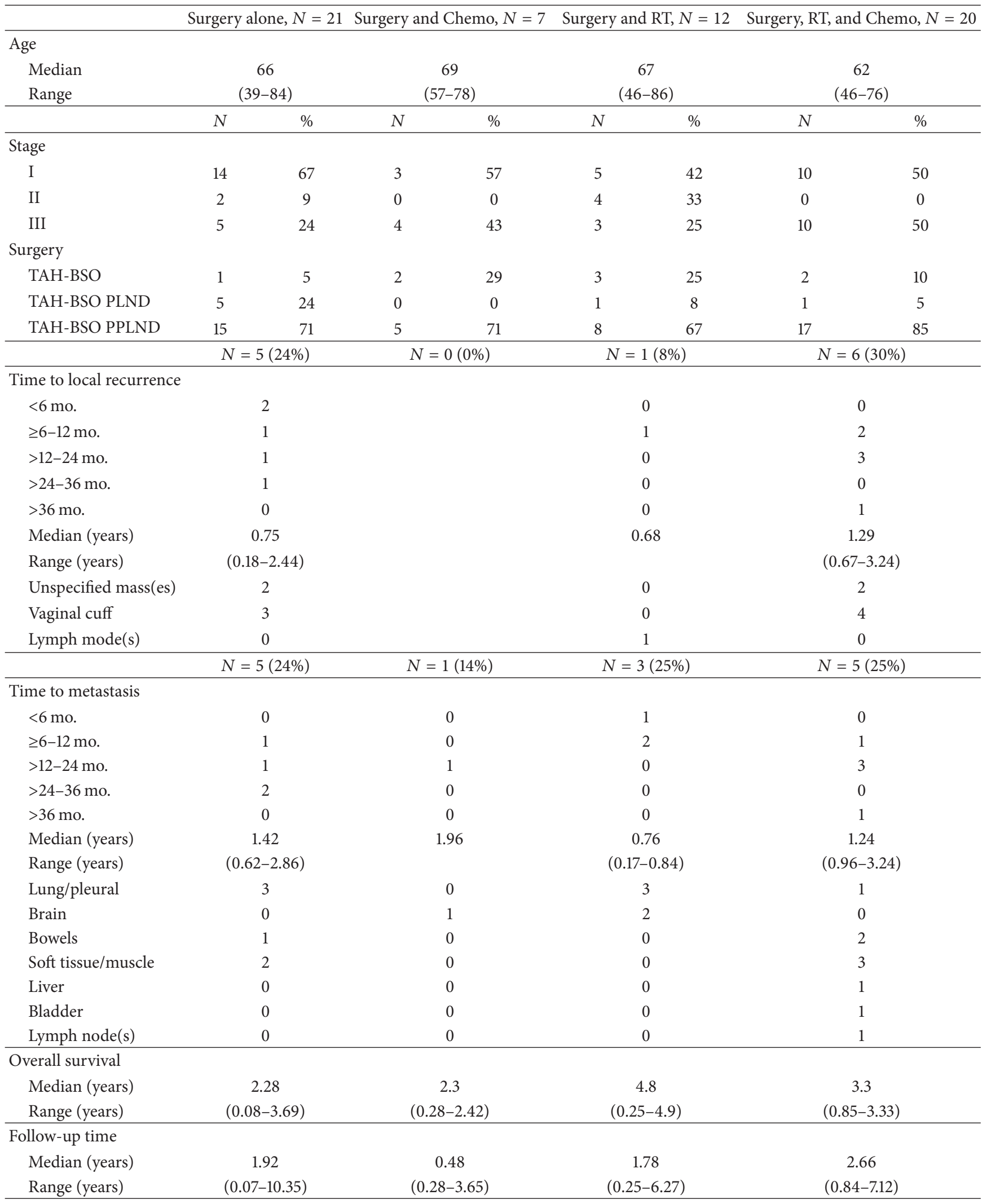


TABLE 2: Patients' characteristics in different radiotherapy groups.

\begin{tabular}{|c|c|c|c|c|c|c|}
\hline \multirow{2}{*}{$\begin{array}{l}\text { Radiation therapy } \\
\text { Age }\end{array}$} & \multicolumn{2}{|c|}{ Brachytherapy and EBRT, $N=10$} & \multicolumn{2}{|c|}{ EBRT only, $N=8$} & \multicolumn{2}{|c|}{ Brachytherapy only, $N=14$} \\
\hline & \multirow{3}{*}{\multicolumn{2}{|c|}{$\begin{array}{c}65 \\
(46-76) \\
\end{array}$}} & & & \multirow{3}{*}{\multicolumn{2}{|c|}{$\begin{array}{c}63 \\
(47-86) \\
\end{array}$}} \\
\hline Median & & & \multirow{2}{*}{\multicolumn{2}{|c|}{$\begin{array}{c}61 \\
(50-76) \\
\end{array}$}} & & \\
\hline \multirow[t]{2}{*}{ Range } & & & & & & \\
\hline & $N$ & $\%$ & $N$ & $\%$ & $N$ & $\%$ \\
\hline \multicolumn{7}{|l|}{ Stage } \\
\hline I & 2 & 20 & 4 & 50 & 9 & 64 \\
\hline II & 2 & 20 & 2 & 25 & 0 & 0 \\
\hline III & 6 & 60 & 2 & 25 & 5 & 36 \\
\hline Chemotherapy & 6 & 60 & 6 & 75 & 8 & 57 \\
\hline Local recurrence & & $2(20 \%)$ & & $4(50 \%)$ & & $1(7 \%)$ \\
\hline Unspecified mass(es) & & 1 & & 1 & & 0 \\
\hline Vaginal cuff & & 1 & & 2 & & 1 \\
\hline Lymph mode(s) & & 0 & & 1 & & 0 \\
\hline Distant metastases & & $5(50 \%)$ & & $2(25 \%)$ & & $2(14 \%)$ \\
\hline Lung/pleural & & 4 & & 0 & & 1 \\
\hline Brain & & 1 & & 0 & & 1 \\
\hline Bowels & & 2 & & 0 & & 0 \\
\hline Soft tissue/muscle & & 2 & & 2 & & 0 \\
\hline Liver & & 0 & & 1 & & 0 \\
\hline Bladder & & 1 & & 0 & & 0 \\
\hline Lymph node(s) & & 0 & & 1 & & 0 \\
\hline Radiation toxicity & & $8(80 \%)$ & & $6(75 \%)$ & & $2(14 \%)$ \\
\hline Grade 1-2 & & 7 & & 3 & & 1 \\
\hline Grade 3-4 & & 1 & & 3 & & 1 \\
\hline
\end{tabular}

Pairwise comparisons showed there is no stage II difference between other group to group comparisons. There was a statistically significant difference of stage I patients between the EBRT and brachytherapy group and the brachytherapy only group $(P=0.01)$. Global tests showed no difference in the distribution among the groups for stage II $(P=0.13)$ or stage III $(P=0.66)$ or differences in stage I distribution between EBRT only and brachytherapy only $(P=0.42)$ or EBRT only and EBRT plus brachytherapy $(P=0.12)$.

In this study, 9 out of 21, 3 out of 7,7 out of 12 , and 12 out of 20 patients survived following surgery alone, surgery plus chemotherapy, surgery plus radiation therapy, and a combination of surgery, chemotherapy, and radiation therapy groups, respectively. The estimated 1-year OS and 3-year OS for those who had surgery alone were $71 \%$ and $44 \%$, respectively. The 1-year OS and 3-year OS were estimated to be $91 \%$ and $61 \%$ for those who had surgery with radiotherapy. Patients who had surgery plus chemotherapy had a calculated 1 -year OS of $57 \%$ and a calculated 3 -year OS of $28 \%$. In addition, 1-year OS and 3-year OS are $90 \%$ and $62 \%$, respectively, in the trimodality treatment group. Moreover, the 1-year, 3-year, and 5-year overall survival for all 60 patients during the past 12 years are $80 \%, 53 \%$, and $43 \%$, respectively. The 5-year overall survival of all patients encountered our institution including stage IV disease is $36 \%$.
With an average follow-up time of 2.8 years, 12 patients developed local recurrence and 14 patients developed distant failure. Median time to local recurrence and median time to distant metastases were 0.99 years and 1.27 years, respectively. True median time to local recurrence and median time to distant metastases cannot be calculated here as less than $50 \%$ of the population experienced the event. For these 60 patients, the overall 1-year local recurrence rate (LRR) is $11.2 \%$ and the 3-year LRR is $24.7 \%$. The 1-year and 3-year distant recurrence rates are $11.6 \%$ and $23.4 \%$, respectively.

Further data analysis showed that the combination of surgery plus chemotherapy plus radiation therapy and surgery plus radiation therapy showed significant improvement of OS compared to surgery alone $(P=0.044$ and 0.028 , resp.) (Figure 1(a)). Not counting the patient who received surgery 179 days after diagnosis, overall survival comparison remains at a statistically significant level $(P=0.03$ and 0.015 , resp.).

Our data suggests that trimodality therapy with surgery plus chemotherapy plus radiation therapy and surgery plus radiation therapy likely improves OS compared to surgery plus chemotherapy. However, due to the limited number of patients in each group, this did not reach statistical significance $(P=0.12$ and 0.093 , resp.) (Figure $1(\mathrm{~b}))$. Adding radiation to surgery improved median survival from 2.3 years 


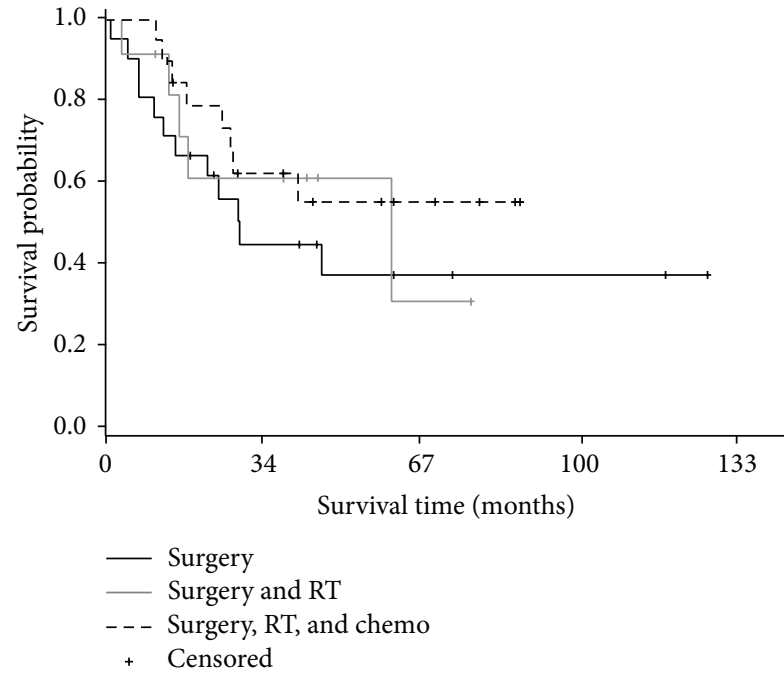

(a)

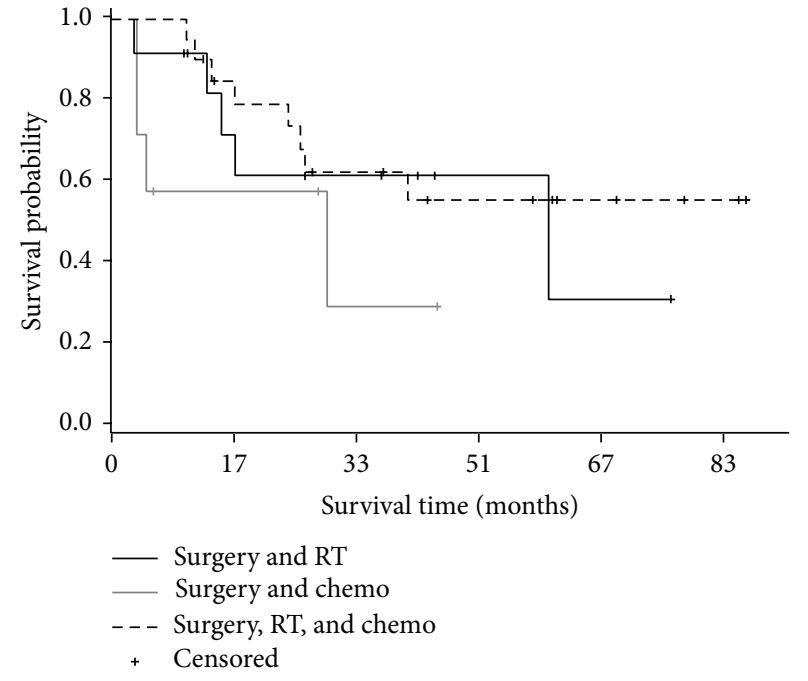

(b)

FIGURE 1: All stages, overall survival by treatment. (a) Surgery alone versus surgery plus radiotherapy $(P=0.028)$; surgery alone versus surgery plus chemoradiation therapy $(P=0.044)$. (b) Surgery plus chemotherapy versus surgery plus radiotherapy $(P=0.093)$; surgery plus chemotherapy versus surgery plus chemoradiation therapy $(P=0.12)$.

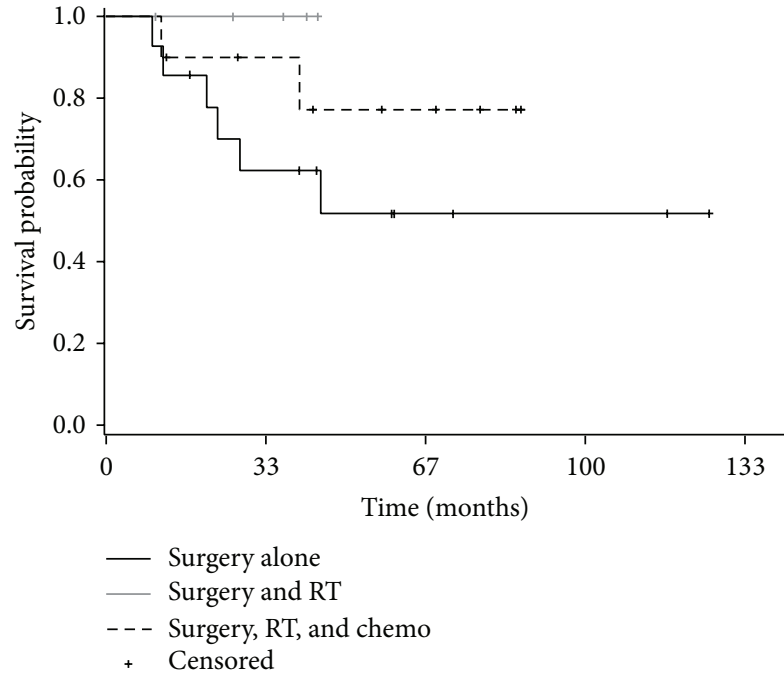

Figure 2: Surgical stage I, overall survival by treatment. Surgery alone versus surgery plus radiotherapy $(P=0.24)$.

to 4.8 years with an average follow-up time of 2.8 years. It is worthwhile to note that there was a possible improvement in overall survival with the addition of radiation therapy in stage I patients compared to surgery alone with a hazard ratio of $0.424(P=0.24)$ (Figure 2$)$. In stage II and III patients, there were no discernible overall survival differences among the three groups (surgery versus surgery plus RT, $P=0.82$; surgery versus trimodality, $P=0.94$; surgery plus RT versus trimodality, $P=0.75$ ).

In addition to overall survival, we also compared local recurrence and distant metastasis free survival among all three groups ( $P=0.49$ and $P=0.99$, resp. $)$. There were no

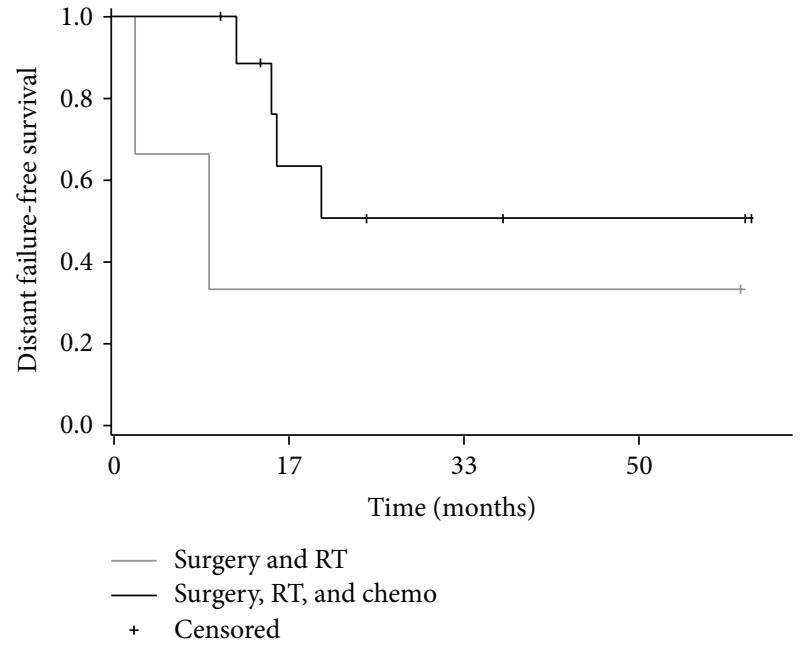

FIGURE 3: Surgical stage III, distant metastasis by treatment. Surgery plus radiotherapy versus surgery plus chemoradiation therapy $(P=$ $0.23)$.

significant differences identified. In stage III patients, those who received trimodality therapy had less distant metastases compared to the surgery plus radiotherapy group $(P=$ 0.23 ) (Figure 3). Among stage III patients in the trimodality group, 2/10 received EBRT only, 4/10 received both EBRT and brachytherapy, and 4/10 received brachytherapy alone. Among patients in the surgery plus RT group, 2/3 received EBRT plus brachytherapy, and $1 / 3$ received brachytherapy only.

We compared different radiation therapy modalities in the group of surgery plus radiation and the group of surgery plus chemoradiation. Patients in the brachytherapy alone 


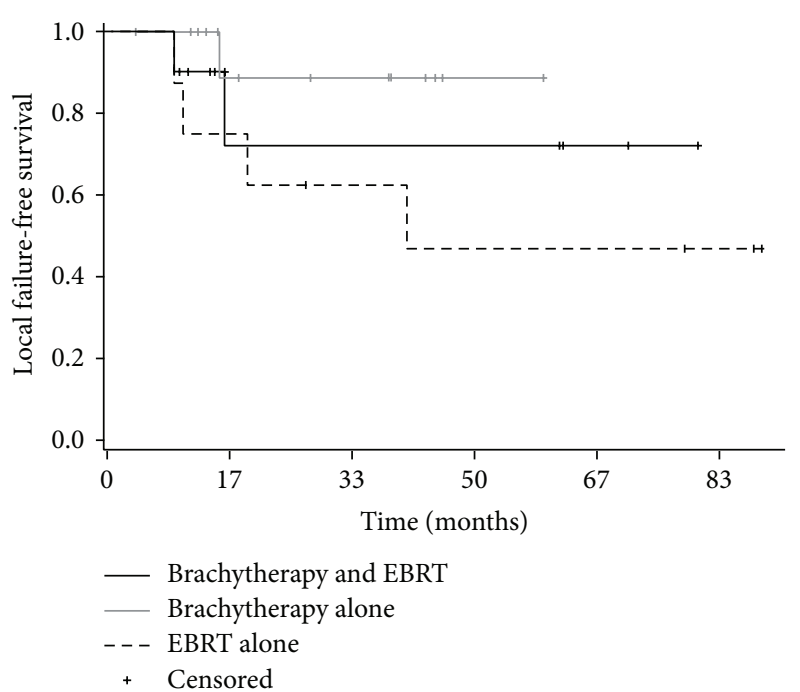

(a)

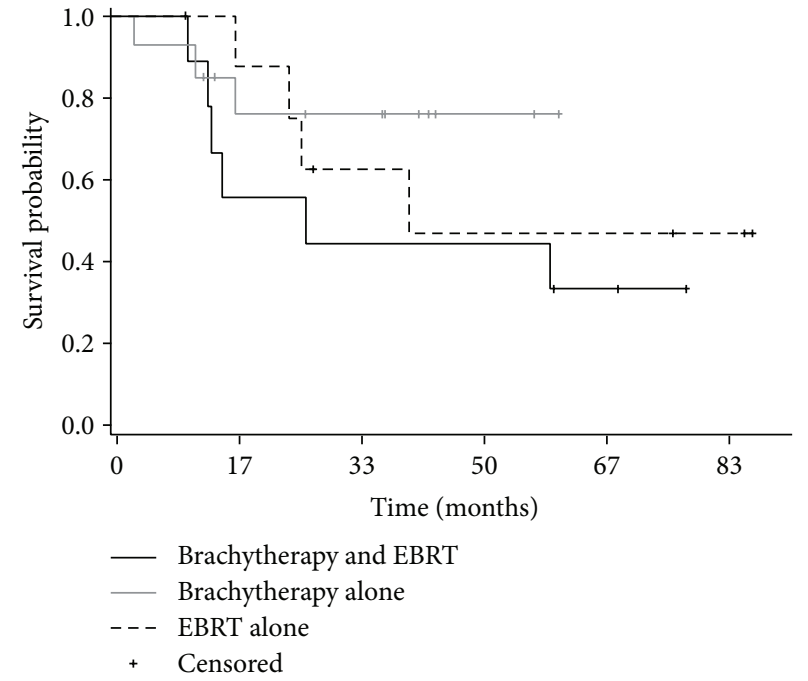

(b)

FIGURE 4: (a) All stages, local recurrence by radiotherapy. Brachytherapy only versus EBRT only $(P=0.08)$. (b) All stages, overall survival by radiotherapy. Brachytherapy only versus EBRT only $(P=0.81)$. Brachytherapy only versus EBRT plus brachytherapy $(P=0.32)$. EBRT plus brachytherapy versus EBRT only $(P=0.68)$.

group showed equally effective decreased local recurrence rate (LRR) compared to the group that received EBRT alone $(P=0.08)$ (Figure $4(\mathrm{a}))$. Only one patient (stage III) in the brachytherapy alone group developed local recurrence. However, the LRR difference between brachytherapy alone and EBRT alone did not reach statistical significance in our study. Similarly, no local recurrence differences were found when comparing both brachytherapy and EBRT versus brachytherapy alone $(P=0.63)$ and both versus EBRT alone $(P=0.68)$. There was also no significant difference when comparing distant recurrence among different radiation modalities. Overall survival was not significantly longer in any of these 3 different radiation therapy modalities (Figure 4(b)). We further grouped EBRT alone and EBRT plus brachytherapy together and analyzed their local recurrence, distant recurrence, and overall survival as compared to the brachytherapy alone group. No significant differences were detected $(P=0.15,0.32$, and 0.24 , resp.). There was also no statistically significant distribution difference between stages I, II, and III of these two groups $(P=0.07,0.11$, and 0.49 resp.).

Adverse side effects were analyzed among different radiation regimens and graded according to Common Terminology Criteria for Adverse Events (CTCAE) v.4.0. In patients who received EBRT, grade 1-2 side effects with the most common being GI irritations such as diarrhea and rectal urgency were noted in ten out of 18 patients (56\%). Four out of 18 patients developed grade 3-4 side effects (22\%). Three patients developed small bowel obstruction (SBO) and one of them underwent surgical resection. One patient developed severe enteritis, for which she had intestinal resection. One patient in the brachytherapy only $(n=14)$ group developed mild diarrhea (7\%). Another patient developed partial SBO (small bowel obstruction), which is unlikely, but might be related to radiation (Table 2). In twelve patients that received chemotherapy in addition to EBRT, 7 of them developed grade 1 - 2 side effects (58\%) and 2 of them developed grade 3 4 side effects (17\%). In brachytherapy alone group, 8 patients received chemotherapy, of which 1 developed grade $1-2$ side effects (13\%) and 1 developed grade 3-4 side effects (13\%).

The most common surgical complications in these patients are wound dehiscence (5 patients), incisional hernia (3 patients), and postoperative ileus (3 patients).

\section{Discussion}

Our single-institution findings demonstrate that $15 \%$ of surgically staged uterine carcinosarcomas presented with distant metastasis, $43 \%$ presented with disease outside of the uterus, and only $43 \%$ presented with stage I disease. These numbers are comparable with previous studies given different patient populations and surgical techniques $[2,14,15]$. According to our data, adjuvant radiation and chemotherapy or radiation and chemotherapy alone offered overall survival advantages in carcinosarcoma of all stages. Although surgery alone was performed in relatively early-stage group of patients, it has an inferior overall survival compared to more advancedstage patients who were treated with adjuvant radiation therapy or adjuvant chemoradiation. This suggests that even in stage I carcinosarcoma, surgery alone is not sufficient and adjuvant radiation therapy is beneficial. It is possible that there is occult microscopic disease in early-stage patients. It is also noted that all three combined modalities might have a trend towards an increase in overall survival rates of stage I patients compared to surgery alone. Such a trend was not observed in stage II and III patients. This suggests that adjuvant chemotherapy and radiotherapy is more effective in controlling microscopic disease in early-stage patients. 
Chemotherapy and radiotherapy have demonstrated efficacy for carcinosarcoma patients $[6,12,16-18]$. No statistically significant difference was identified between adjuvant chemotherapy and radiation therapy [19]. A multimodality approach is favored by most institutions. The findings at our institution revealed similar results; there is an overall survival advantage if adjuvant radiotherapy was used, and we failed to detect statistically significant survival benefits for chemotherapy as opposed to radiotherapy. We noted a decline in distant metastatic rate for patients with stage III disease receiving chemotherapy but not in patients with early-stage disease. This is perhaps due to the greater chances of having occult distant metastases upon diagnosis. Administering chemotherapy immediately may limit advanced-stage disease. However, these results should be viewed with caution due to the small sample size and relatively short follow-up time.

Both EBRT and LDR brachytherapy have been reported to promote locoregional free survival $[12,13,20]$. For HDR brachytherapy we used a dose of 5-6 Gy $\times 3$ to limit late side effects [21]. Our data suggests that adjuvant brachytherapy with three HDR vaginal cylinder fractions results in similar local control as compared to EBRT. The high dose delivered by brachytherapy to the local tumor bed may be as effective as EBRT in terms of controlling local recurrence. However, despite our stage distributions there was no statistical significant difference between EBRT only and brachytherapy only. It is possible that physicians selectively recommended brachytherapy only to patients with early-stage disease, which results in superior local control. Although our data analysis between different treatment groups did not reveal significant stage differences, it was limited due to the small sample size in each group. Our data demonstrates (on an admittedly limited scale) that brachytherapy alone is not inferior as compared to the other two groups. Furthermore, patients treated with different radiation modalities demonstrated similar overall survival. This suggests that EBRT over several weeks does not offer additional benefit to carcinosarcoma patients. Three fractions of HDR vaginal cylinder brachytherapy appears to have an equivalent survival benefit as compared to 5 weeks of daily EBRT treatments.

Side effects were noted to be higher with EBRT compared to brachytherapy only. Chemotherapy may also contribute to the side effects. In our study no difference in toxicity was noted between EBRT, with or without chemotherapy. However, in the brachytherapy group, the addition of chemotherapy contributes to the GI side effects.

The median survival of our patients is 24.5 months as calculated from date of diagnosis with a median delay of 24 days between diagnosis and surgery, compared to 16 months reported in other studies [22, 23]. This difference in median survival may be attributed to the fact that we included different patient populations and stages in our study compared to the other studies [24]. Our 5-year overall survival rate is $36 \%$ while the historic 5 -year survival rate is 33-39\%. Thus, the treatment experiences at our institution share parity with other institutions. Our study has two main limitations. Firstly, as with any retrospective study, there may be a potential selection bias with respect to recommendations regarding adjuvant therapy. Secondly, although this is one of the larger reports of uterine carcinosarcoma, subcategorized patient numbers have limited the scope of our analysis and comparison across treatment groups.

Uterine carcinosarcoma is a rare, aggressive malignant tumor with poor prognosis. Multiple treatment modalities published in the literature show impact on local control and overall survival. Surgery plus adjuvant radiation and chemotherapy shows superior survival even in early stages in our study. Nevertheless, a randomized multi-institutional prospective clinical trial is needed to confirm the optimal treatment approach.

\section{Conflict of Interests}

Hualei Li, Mindi J. TenNapel, Amina Ahmed, MD, Lilie Lin, MD, Sudershan K. Bhatia, and Geraldine Jacobson declare that there is no conflict of interests regarding the publication of this paper.

\section{References}

[1] E. D'angelo and J. Prat, "Pathology of mixed Mullerian tumours," Best Practice \& Research Clinical Obstetrics \& Gynaecology, vol. 25, no. 6, pp. 705-718, 2011.

[2] S. E. Brooks, M. Zhan, T. Cote, and C. R. Baquet, "Surveillance, epidemiology, and end results analysis of 2677 cases of uterine sarcoma 1989-1999," Gynecologic Oncology, vol. 93, no. 1, pp. 204-208, 2004.

[3] S. A. El-Nashar and A. Mariani, "Uterine carcinosarcoma," Clinical Obstetrics and Gynecology, vol. 54, no. 2, pp. 292-304, 2011.

[4] S. N. Nielsen, K. C. Podratz, B. W. Scheithauer, and P. C. O’Brien, "Clinicopathologic analysis of uterine malignant mixed Mullerian tumors," Gynecologic Oncology, vol. 34, no. 3, pp. 372-378, 1989.

[5] T. V. Dinh, R. E. Slavin, B. S. Bhagavan, E. V. Hannigan, E. M. Tiamson, and R. B. Yandell, "Mixed Mullerian tumors of the uterus: a clincopathologic study," Obstetrics and Gynecology, vol. 74, no. 3, part 1, pp. 388-392, 1989.

[6] J. Gonzalez Bosquet, S. A. Terstriep, W. A. Cliby et al., "The impact of multi-modal therapy on survival for uterine carcinosarcomas," Gynecologic Oncology, vol. 116, no. 3, pp. 419-423, 2010.

[7] S. E. Ferguson, C. Tornos, A. Hummer, R. R. Barakat, and R. A. Soslow, "Prognostic features of surgical stage I uterine carcinosarcoma," American Journal of Surgical Pathology, vol. 31, no. 11, pp. 1653-1661, 2007.

[8] K. Galaal, F. M. Kew, K. F. Tam et al., "Evaluation of prognostic factors and treatment outcomes in uterine carcinosarcoma," European Journal of Obstetrics Gynecology and Reproductive Biology, vol. 143, no. 2, pp. 88-92, 2009.

[9] M. Callister, L. M. Ramondetta, A. Jhingran, T. W. Burke, and P. J. Eifel, "Malignant mixed Mullerian tumors of the uterus: analysis of patterns of failure, prognostic factors, and treatment outcome," International Journal of Radiation Oncology Biology Physics, vol. 58, no. 3, pp. 786-796, 2004.

[10] G. Sutton, V. L. Brunetto, L. Kilgore et al., "A phase III trial of ifosfamide with or without cisplatin in carcinosarcoma of the uterus: a Gynecologic Oncology Group Study," Gynecologic Oncology, vol. 79, no. 2, pp. 147-153, 2000. 
[11] H. D. Homesley, V. Filiaci, M. Markman et al., "Phase III trial of ifosfamide with or without paclitaxel in advanced uterine carcinosarcoma: a Gynecologic Oncology Group Study," Journal of Clinical Oncology, vol. 25, no. 5, pp. 526-531, 2007.

[12] K. Gerszten, C. Faul, S. Kounelis, Q. Huang, J. Kelley, and M. W. Jones, "The impact of adjuvant radiotherapy on carcinosarcoma of the uterus," Gynecologic Oncology, vol. 68, no. 1, pp. 8-13, 1998.

[13] N. S. Reed, C. Mangioni, H. Malmstrom et al., "Phase III randomised study to evaluate the role of adjuvant pelvic radiotherapy in the treatment of uterine sarcomas stages I and II: an European Organisation for Research and Treatment of Cancer Gynaecological Cancer Group Study (protocol 55874)," European Journal of Cancer, vol. 44, no. 6, pp. 808-818, 2008.

[14] T. S. Pradhan, E. E. Stevens, M. Ablavsky, G. Salame, Y.-C. Lee, and O. Abulafia, "FIGO staging for carcinosarcoma: can the revised staging system predict overall survival?" Gynecologic Oncology, vol. 123, no. 2, pp. 221-224, 2011.

[15] N. Bansal, T. J. Herzog, V. E. Seshan et al., "Uterine carcinosarcomas and grade 3 endometrioid cancers: evidence for distinct tumor behavior," Obstetrics and Gynecology, vol. 112, no. 1, pp. 64-70, 2008.

[16] K. Galaal, K. Godfrey, R. Naik, A. Kucukmetin, and A. Bryant, "Adjuvant radiotherapy and/or chemotherapy after surgery for uterine carcinosarcoma," Cochrane Database of Systematic Reviews, no. 2, Article ID CD006812, 2011.

[17] J. Menczer, T. Levy, B. Piura et al., "A comparison between different postoperative treatment modalities of uterine carcinosarcoma," Gynecologic Oncology, vol. 97, no. 1, pp. 166-170, 2005.

[18] T. Le, "Adjuvant pelvic radiotherapy for uterine carcinosarcoma in a high risk population," European Journal of Surgical Oncology, vol. 27, no. 3, pp. 282-285, 2001.

[19] A. H. Wolfson, M. F. Brady, T. Rocereto et al., "A gynecologic oncology group randomized phase III trial of whole abdominal irradiation (WAI) vs. cisplatin-ifosfamide and mesna (CIM) as post-surgical therapy in stage I-IV carcinosarcoma (CS) of the uterus," Gynecologic Oncology, vol. 107, no. 2, pp. 177-185, 2007.

[20] T. P. Manolitsas, G. V. Wain, K. E. Williams, M. Freidlander, and N. F. Hacker, "Multimodality therapy for patients with clinical Stage I and II malignant mixed Mullerian tumors of the uterus," Cancer, vol. 91, no. 8, pp. 1437-1443, 2001.

[21] S. Nag, B. Erickson, S. Parikh, N. Gupta, M. Varia, and G. Glasgow, "The American Brachytherapy Society recommendations for high-dose-rate brachytherapy for carcinoma of the endometrium," International Journal of Radiation Oncology Biology Physics, vol. 48, no. 3, pp. 779-790, 2000.

[22] A. L. Jonson, R. L. Bliss, A. Truskinovsky et al., "Clinical features and outcomes of uterine and ovarian carcinosarcoma," Gynecologic Oncology, vol. 100, no. 3, pp. 561-564, 2006.

[23] A. Gadducci, E. Sartori, F. Landoni et al., "The prognostic relevance of histological type in uterine sarcomas: a cooperation task force (CTF) multivariate analysis of 249 cases," European Journal of Gynaecological Oncology, vol. 23, no. 4, pp. 295-299, 2002.

[24] C. D. Arrastia, R. G. Fruchter, M. Clark et al., "Uterine carcinosarcomas: incidence and trends in management and survival," Gynecologic Oncology, vol. 65, no. 1, pp. 158-163, 1997. 


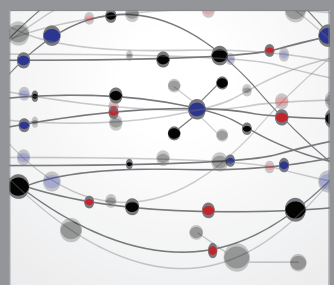

The Scientific World Journal
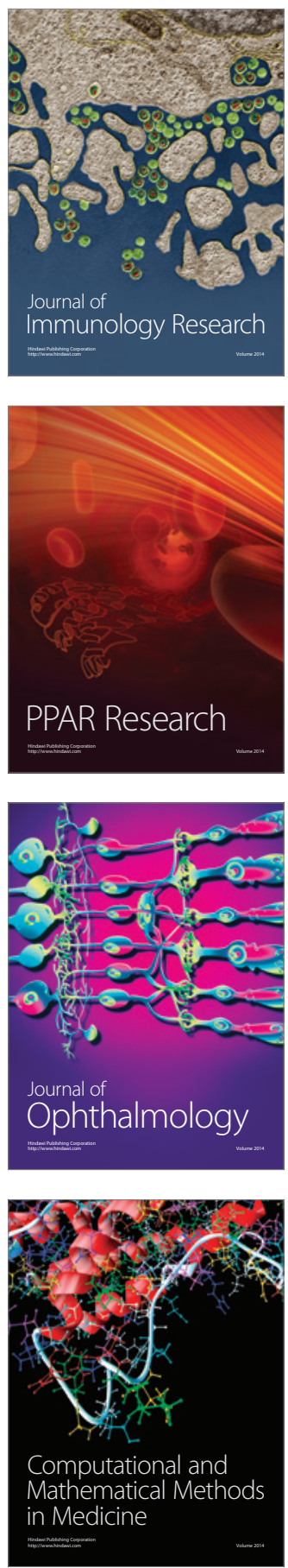

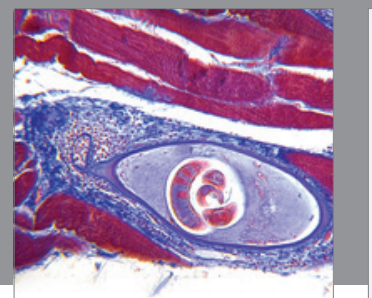

Gastroenterology

Research and Practice
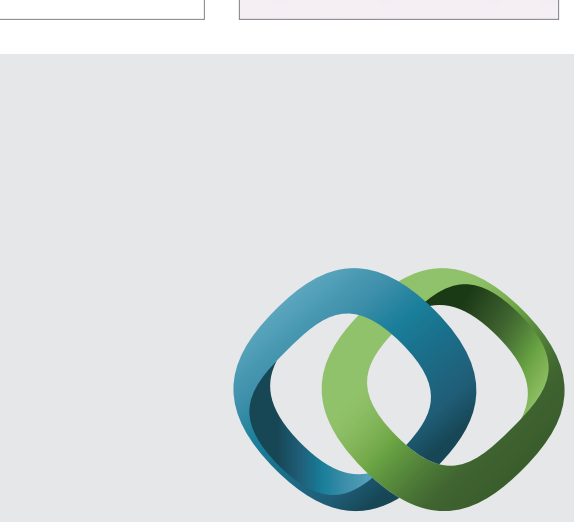

\section{Hindawi}

Submit your manuscripts at

http://www.hindawi.com
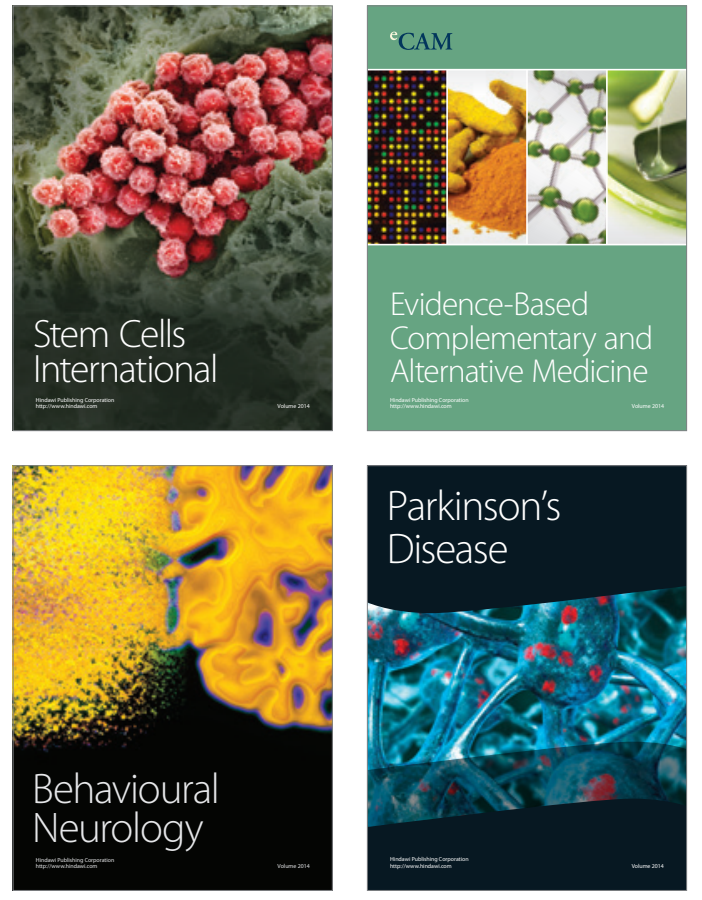
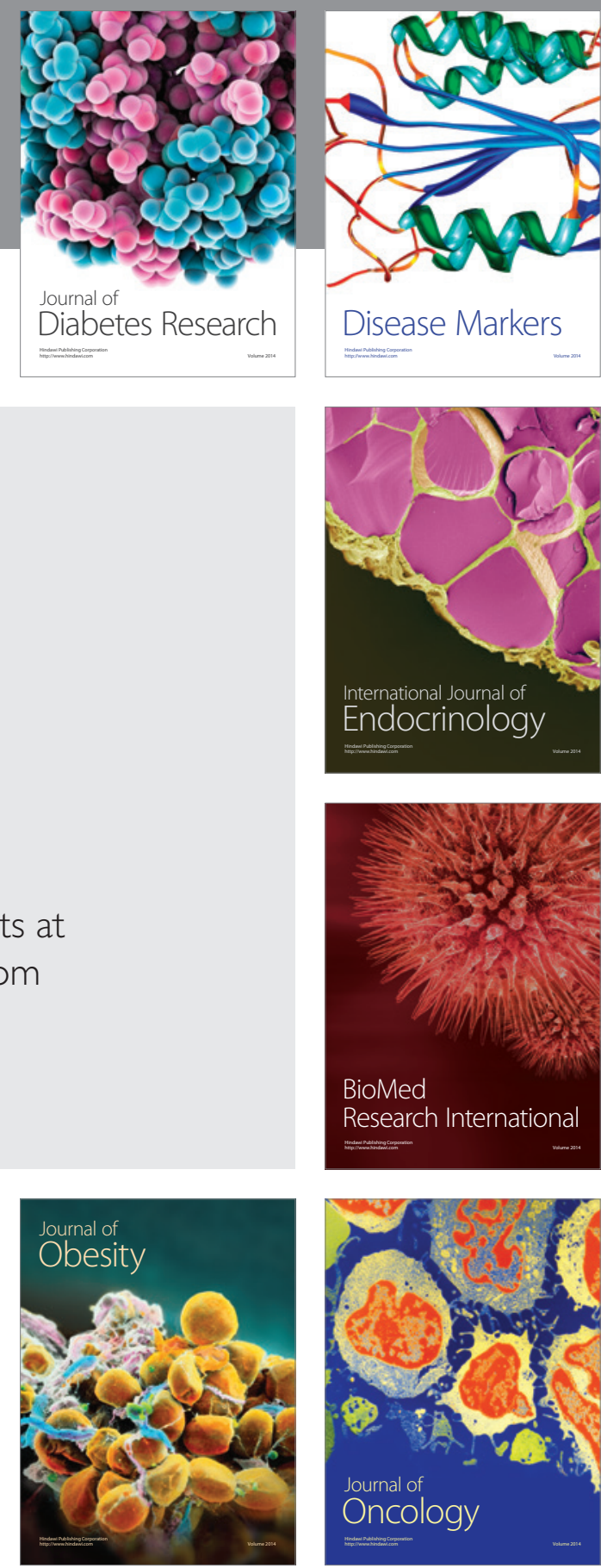

Disease Markers
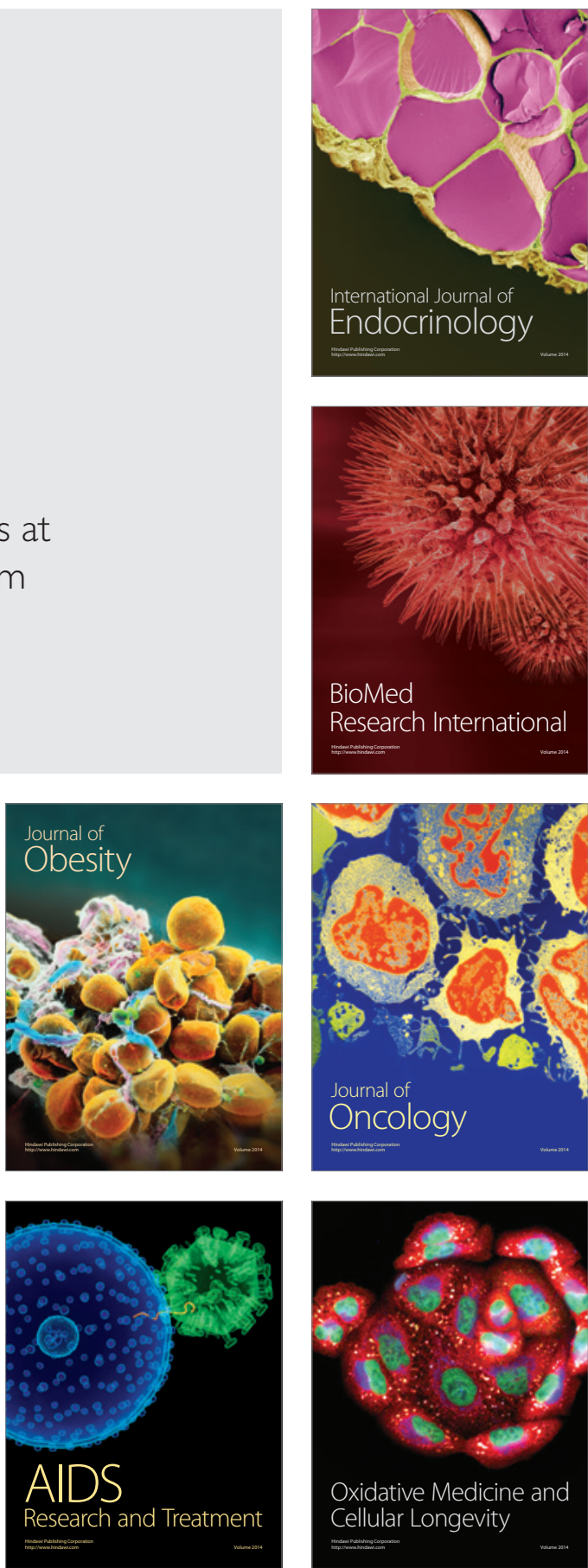